\title{
Correlação entre equilíbrio e ambiente domiciliar como risco de quedas em idosos com acidente vascular encefálico
}

Correlation between balance and home environment to risk of falls of elderly with stroke

Priscila Santos Borges*

Luiz Evandro Nunes Marinho Filho* Cláudio Henrique Meira Mascarenhas*
Resumo

Introdução: Os idosos acometidos pelo acidente vascular encefálico (AVE) com presença de alterações de equilíbrio estão mais suscetíveis a quedas, podendo estar envolvidos fatores de riscos ambientais em sua etiologia. Objetivo: Correlacionar o equilíbrio e o ambiente domiciliar como risco de quedas em idosos acometidos pelo AVE. Metodologia: Estudo descritivo, transversal, quantitativo, cuja amostra foi de 25 idosos, com 60 anos ou mais, de ambos os sexos, com história prévia de AVE, residentes na comunidade e atendidos em dois centros e duas unidades de saúde do município de Jequié$\mathrm{BA}$, selecionados intencionalmente. Resultados: $\mathrm{O}$ grupo estudado apresentou uma média de 28,96 $( \pm 15,65)$ segundos no Timed Up and Go Test, variando de 8 a 70 segundos, sendo que $36 \%$ dos indivíduos realizaram o teste em 30 segundos ou mais, apresentando alto risco de quedas. Na Escala Ambiental de Risco de Quedas Adaptada, os domicílios avaliados apresentaram média de 20,12 ( \pm $4,79)$ pontos, variando de 9 a 27 pontos, em que se verificou que 56\% apresentaram pontuação maior que 20 , demonstrando alto risco de quedas para os idosos. A correlação entre o equilíbrio dos idosos acometidos pelo AVE com o ambiente domiciliar mostrou-se positiva e estatisticamente significativa $(\mathrm{p}=0,039)$ Conclusão: A dificuldade de equilíbrio apresentada pelos idosos com AVE associada à alta prevalência de fatores de riscos encontrados no ambiente domiciliar remete a um quadro preocupante, tornando-se necessária a criação de medidas que visem à prevenção das quedas, com o intuito de melhorar a qualidade de vida dessa população.

\footnotetext{
Universidade Estadual do Sudoeste da Bahia, Departamento de Saúde, Curso de Fisioterapia. Jequié, BA, Brasil.
}

Palavras-chave: Idoso. Acidente Cerebral Vascular. Equilíbrio Postural. Acidentes por Quedas. Fatores de Risco. Riscos Ambientais. Etiologia. Saúde do Idoso. 


\section{Abstract}

Introduction: Elderly who suffer from stroke with balance alterations are more likely to fall, and environmental risk factors may be involved in its etiology. Objective: To correlate balance and home environment to risk of falls of elderly with stroke. Methods: It's a descriptive, quantitative, cross-sectional study, and the sample comprised 25 elderly, aged 60 years or more, of both sexes, with previous history of stroke, living in the community and attended in two centers and two health units in the city of Jequié, State of Bahia, intentionally selected. Results: The studied group presented a mean of $28.96( \pm 15.65)$ seconds on the Timed Up and Go Test, varying from 8 to 70 seconds, and $36 \%$ of the individuals did the test in 30 seconds or more, presenting high risk of falls. On the Environmental Risk of Falls Adapted Scale, the evaluated houses showed a mean of $20.12( \pm 4.79)$ points, varying from 9 to 27 points, where it was verified that $56 \%$ of the elderly had score higher than 20, showing high risk of falls among the elderly. The correlation between the balance of the elderly with stroke and home environment was positive and statistically significant $(p=0,039)$. Conclusion: The difficulty of balance showed by the elderly with stroke associated to high prevalence of risk factors found at home environment cross-refers to a worrying situation, and it becomes necessary to create actions towards the prevention of falls, with the intention of improving the quality of life of that population.
Key words: Aged. Stroke. Postural Balance. Accidental Falls. Risk Factors. Environmental Risks. Etiology. Health of the Elderly.

\section{INTRODUÇÃO}

O envelhecimento populacional deixou de ser um fenômeno característico de países desenvolvidos para tornar-se eminente em países como o Brasil, cuja tendência é possuir uma das maiores populações compostas por idosos do mundo., ${ }^{1,2}$ Estima-se que em 2020, os idosos constituirão $13 \%$ da sociedade brasileira, correspondendo a 13,5 milhões de indivíduos. ${ }^{1,3}$

O aumento da proporção de idosos juntamente com o crescimento da longevidade trazem mudanças ao panorama epidemiológico de uma população. As doenças infecto-contagiosas mais prevalentes em indivíduos jovens diminuem progressivamente, enquanto ascendem as patologias crônico-degenerativas mais incidentes na população geriátrica. ${ }^{4}$

Dentre as doenças crônico-degenerativas, o acidente vascular encefálico (AVE) pode ser destacado por contribuir com cerca de 5 milhões de mortes e mais de 15 milhões de casos não fatais anualmente, sendo a segunda causa de morte em todo o mundo. ${ }^{5}$ Paralelamente aos altos índices de morbi-mortalidade, a situação é agravada pela elevada probabilidade de um evento recidivante, sendo que cada episódio constitui alto risco de mortalidade, incapacidade e dependência permanente. $^{6}$

Depois de um episódio de AVE, pelo menos dois terços dos sobreviventes apresentam algum grau de deficiência decorrente dos déficits neurológicos instalados. ${ }^{1,3}$ As sequelas geradas, tais como contraturas e deformidades que resultam na perda de movimentos, espasticidade e posicionamento impróprio, alteram a biomecânica articular normal, provocando alterações no equilíbrio e instabilidade postural, com consequente risco para quedas.?

Os idosos que apresentam história prévia de AVE com presença de alterações de mobilidade, equilíbrio e controle postural estão mais suscetíveis a eventos de quedas, sendo sua incidência diretamente proporcional ao grau de déficit funcional do indivíduo. ${ }^{8}$

A lesão acidental é a sexta causa de mortalidade em pessoas de 75 anos ou mais, ${ }^{9}$ sendo a queda a principal etiologia em indivíduos maiores de 65 anos. ${ }^{9}, 10$ Aproximadamente $30 \%$ dos idosos em 
países ocidentais sofrem queda ao menos uma vez ao ano, sendo que, destes, cerca de $50 \%$ sofrem duas ou mais. ${ }^{4,11}$

A queda e suas consequências podem estar presentes em todas as épocas da vida; entretanto, para os idosos, elas possuem significado muito relevante, pois podem gerar possíveis complicações, como fraturas, medo de cair, restrição de atividades da vida diária, declínio da saúde, aumento do risco de institucionalização, incapacidade e morte. ${ }^{9,11,12}$

Diversos fatores de risco podem ser potenciais agentes determinantes de quedas, e a probabilidade desse evento aumenta à medida que estes fatores se acumulam. Os mesmos podem ser dispostos em dois grupos: intrínsecos, que dizem respeito às características inerentes ao indivíduo, como alterações de mobilidade e equilíbrio decorrentes do envelhecimento, doenças crônicas e efeitos adversos de medicamentos em uso; e extrínsecos, que incluem perigos ambientais, bem como o tipo de atividade exercida. ${ }^{12}$

A prevalência de queixas de equilíbrio na população acima de 65 anos chega a $85 \%$, podendo estar associada a diversas etiologias e manifestando-se por sinais e sintomas, como tontura, vertigem, desequilíbrio, desvio de marcha, instabilidade, náuseas e quedas frequentes. ${ }^{13}$

Durante o processo de envelhecimento, o feedback para os centros de controle postural tornase reduzido ou inapropriado. Concomitantemente, o sistema músculo-esquelético é acometido, podendo perder a capacidade de responder de forma adequada aos distúrbios da estabilidade postural. ${ }^{8}$

Além dos fatores intrínsecos, estudos demonstram que uma porcentagem significativa das quedas ocorre no ambiente domiciliar dos idosos, ${ }^{14}$ e nos cômodos mais utilizados, ${ }^{15}$ sendo provocada por fatores de risco do ambiente físico durante o exercício das atividades da vida diária.? Com a presença de tais fatores predisponentes, aumenta a probabilidade de eventos como escorregões, tropeços, trombadas, erros no passo, constituindo risco para os idosos ativos. Em idosos acometidos pelo AVE com comprometimento do equilíbrio, o risco apresentado seria acentuado. ${ }^{7}$

Embora seja evidente o aumento dos eventos de quedas entre idosos, a literatura gerontológica e geriátrica brasileira tem efetuado poucos estudos epidemiológicos relacionando a história de quedas em pacientes portadores de distúrbios neurológicos como o AVE, bem como os fatores envolvidos na sua etiologia. Dessa forma, este estudo propõe correlacionar o equilíbrio e o ambiente domiciliar para risco de quedas em idosos acometidos pelo AVE.

\section{METODOLOGIA}

Trata-se de um estudo descritivo, transversal, quantitativo, cuja amostra foi composta por 25 idosos, de ambos os sexos, com história prévia de AVE, residentes na comunidade e atendidos em dois centros e duas unidades de saúde do município de Jequié/BA, selecionados de forma intencional. Considerou-se idoso todo indivíduo com 60 anos ou mais, conforme preconizado pela $\mathrm{OMS}^{16}$ para países em desenvolvimento. ${ }^{17}$

A população de idosos usuários dos centros e unidades de saúde consistiu de 465 indivíduos, sendo que, destes, 38 apresentavam história de AVE segundo informações dos Agentes Comunitários de Saúde (ACS). No entanto, 13 sujeitos foram excluídos do estudo em função dos critérios de exclusão supracitados.

Todos os idosos com história de AVE atendidos nos locais supracitados que aceitaram participar voluntariamente do estudo foram avaliados, independentemente do tempo de acometimento pela patologia. Foram excluídos do estudo os pacientes incapazes de se levantar e manter postura ereta sem auxílio, incapazes de realizar marcha de curtas distâncias de forma independente, que realizassem marcha com auxílio de órteses, portadores de deficiência visual (cegueira) e com déficit de compreensão que limite a execução de movimentos através de comandos verbais. 
Durante o processo de seleção da amostra, foi realizado contato com os agentes comunitários de saúde (ACS) dos centros e unidades de saúde previamente selecionados, através de uma reunião. A partir das informações obtidas em registros dos ACS, foi confeccionada uma lista nominal de pacientes idosos com história prévia de AVE atendidos nos locais anteriormente citados. Em posse dessa lista, foi realizada visita domiciliar aos idosos para que fosse efetuada a coleta de dados do presente estudo.

A coleta de dados foi realizada durante uma visita ao ambiente domiciliar dos idosos participantes do estudo. Inicialmente, foram coletados dados pessoais através de um questionário sócio-demográfico, contendo as seguintes variáveis: faixa etária, sexo, situação conjugal, escolaridade, arranjo familiar e renda. Em seguida, foram realizadas avaliações do equilíbrio dos idosos e do ambiente domiciliar. As entrevistas foram realizadas com os idosos, e as informações confirmadas por um familiar/cuidador.

Para avaliar o equilíbrio, foi utilizado um teste de confiabilidade reconhecida pela literatura, denominado Timed $\mathrm{Up}_{\mathrm{p}}$ and Go (TUG) ${ }^{18}$ desenvolvido com base em um teste já existente, Get $\mathrm{Up}$ and Go, ${ }^{19}$ na tentativa de quantificar o desempenho da mobilidade através $\mathrm{da}$ velocidade do idoso ao realizar a tarefa. $\mathrm{O}$ tempo gasto para completar o teste está fortemente relacionado ao nível de capacidade funcional. ${ }^{12}$

Nesse teste é analisado o tempo gasto pelo indivíduo para partir de uma posição inicial sentada, com as costas apoiadas em uma cadeira, sem o auxílio dos membros superiores, andar um percurso linear de três metros até um ponto predeterminado marcado no chão, girar $180^{\circ}$, retornar e sentar novamente, apoiando as costas na mesma cadeira. O paciente é instruído a realizar o teste usando seus calçados habituais, a não conversar durante a execução e a realizá-lo numa velocidade normal, de forma segura. Maiores valores de tempo representam maiores riscos de queda. ${ }^{18}$
Foram realizados dois testes com dois minutos de intervalo entre os mesmos, sendo que o primeiro teve fins de aprendizado e o segundo serviu como fonte de dados para o estudo. $\mathrm{O}$ tempo foi cronometrado por um relógio digital da marca Condor, modelo B2B, manuseado por um único pesquisador.

Os resultados do TUG foram classificados segundo Podsiadlo \& Richardson, ${ }^{18}$ da seguinte forma: baixo risco de quedas para menos de 20 segundos de realização do teste, médio risco de quedas de 20 a 29 segundos, e alto risco de quedas para 30 segundos ou mais.

Os fatores ambientais foram identificados através da Escala Ambiental de Risco de Quedas Adaptada, ${ }^{19,20}$ que contém questões abordando a segurança de áreas de locomoção, disposição da mobília, iluminação, disponibilidade e acesso aos objetos, nos seguintes locais: quarto de dormir, banheiro, cozinha, escada e sala. O teste consta de 29 questões, sendo realizada uma pontuação no estudo da seguinte forma: "0" para cada resposta afirmativa e "1" para cada negativa, sendo que quanto maior o escore final maior o risco de quedas. Dessa forma, os idosos foram classificados em três grupos de acordo com o escore obtido na escala: menos de 10 pontos foi considerado de baixo risco, de 10 a 20 pontos médio risco e maior que 20 pontos, alto risco ambiental para quedas.

Esta pesquisa obedeceu às normas éticas da Resolução n ${ }^{\circ}$ 196/96 do Conselho Nacional de Saúde. ${ }^{21} \mathrm{O}$ presente estudo foi aprovado pelo Comitê de Ética em Pesquisa da Universidade Estadual do Sudoeste da Bahia (parecer no 074/ 2007) e todos os participantes assinaram o Termo de Consentimento Livre e Esclarecido.

Os dados foram analisados através de estatística descritiva e a relação entre TUG e Escala Ambiental de Risco de Quedas Adaptada foi realizada por meio de Correlação de Spearman utilizando o software SAS (1999), sendo considerado o nível de significância de $5 \%(p<0,05) .{ }^{22}$ 


\section{RESULTADOS}

Foram avaliados 30 idosos com AVE, sendo que, destes, três não participaram do estudo por serem acamados e dois por não conseguirem deambular sem auxílio de órteses, totalizando, ao final da amostra, 25 idosos. A média de idade foi de 72,08 $( \pm 9,22)$ anos, variando de 60 a 94 anos.

Foi verificado que $60 \%$ dos idosos acometidos por AVE eram do sexo feminino, $40 \%$ encontravam-se na faixa etária entre 70 e 79 anos, $44 \%$ eram viúvos, $72 \%$ possuíam nível fundamental incompleto, $48 \%$ moravam com duas gerações e $64 \%$ apresentaram uma renda que variou entre acima de um até dois salários mínimos (tabela 1$)$.

Tabela 1 - Distribuição dos idosos com AVE de acordo com as variáveis sócio-demográficas. Jequié, BA, 2008.

\begin{tabular}{|c|c|c|}
\hline Variáveis & $\mathrm{n}$ & $\%$ \\
\hline \multicolumn{3}{|l|}{ Sexo } \\
\hline Masculino & 10 & 40,0 \\
\hline Feminino & 15 & 60,0 \\
\hline Total & 25 & 100,0 \\
\hline \multicolumn{3}{|l|}{ Faixa etária } \\
\hline $60-69$ anos & 9 & 36,0 \\
\hline 70-79 anos & 10 & 40,0 \\
\hline$\geqslant 80$ anos & 6 & 24,0 \\
\hline Total & 25 & 100,0 \\
\hline \multicolumn{3}{|l|}{ Situação conjugal } \\
\hline Solteiro & 4 & 16,0 \\
\hline Casado & 8 & 32,0 \\
\hline Viúvo & 11 & 44,0 \\
\hline Divorciado & 2 & 8,0 \\
\hline Total & 25 & 100,0 \\
\hline \multicolumn{3}{|l|}{ Escolaridade } \\
\hline Analfabeto & 6 & 24,0 \\
\hline Fundamental Incompleto & 18 & 72,0 \\
\hline Fundamental Completo & 1 & 4,0 \\
\hline Total & 25 & 100,0 \\
\hline \multicolumn{3}{|l|}{ Arranjo familiar } \\
\hline Mora com 1 geração & 2 & 8,0 \\
\hline Mora com 2 gerações & 12 & 48,0 \\
\hline Mora com 3 gerações & 10 & 40,0 \\
\hline Sozinho & 1 & 4,0 \\
\hline Total & 25 & 100,0 \\
\hline \multicolumn{3}{|l|}{ Renda } \\
\hline$\leqslant 1$ salário & 8 & 32,0 \\
\hline $1<\mathrm{x} \leqslant 2$ salários & 16 & 64,0 \\
\hline$>2$ salários & 1 & 4,0 \\
\hline Total & 25 & 100,0 \\
\hline
\end{tabular}

Fonte: Dados da pesquisa, 2008.
Quanto à realização do Timed Up and Go Test (TUG), o grupo estudado apresentou uma média de 28,96 ( $\pm 15,65)$ segundos, variando de 8 a 70 segundos. A partir dos resultados do teste TUG, verificou-se que $36 \%$ dos idosos realizaram o teste em 30 segundos ou mais, apresentando alto risco de quedas (tabela 2).

Tabela 2 - Distribuição dos idosos com AVE de acordo com o Timed Up and Go Test (TUG). Jequié, BA, 2008.

\begin{tabular}{lcc}
\hline Timed Up and Go Test & $\mathrm{n}$ & $\%$ \\
\hline$<20$ s (baixo risco) & 8 & 32 \\
Entre 20 e 29 s (médio risco) & 8 & 32 \\
$\geqslant 30$ s (alto risco) & 9 & 36 \\
Total & 25 & 100 \\
\hline
\end{tabular}

Fonte: Dados da pesquisa, 2008.

$\mathrm{Na}$ Escala Ambiental de Risco de Quedas Adaptada, os domicílios avaliados apresentaram uma média de 20,12 $( \pm 4,79)$ pontos, variando de 9 a 27 pontos. A partir desses dados, pôde-se verificar que $56 \%$ dos domicílios apresentaram pontuação maior que 20 , demonstrando alto risco de quedas para os idosos (tabela 3 ).

Tabela 3 - Distribuição dos idosos com AVE de acordo com a Escala Ambiental de Risco de Quedas Adaptada. Jequié, BA, 2008.

\begin{tabular}{lrr}
\hline Escala Ambiental de Risco de Quedas Adaptada & $\mathrm{n}$ & $\%$ \\
\hline$<10$ pontos (baixo risco) & 1 & 4 \\
Entre 10 e 20 pontos (médio risco) & 10 & 40 \\
$>20$ pontos (alto risco) & 14 & 56 \\
Total & 25 & 100 \\
\hline
\end{tabular}

Fonte: Dados da pesquisa, 2008

Entre os itens analisados na Escala Ambiental de Risco de Quedas Adaptada, pôde-se observar que a cozinha e a sala não apresentaram riscos ambientais significativos (Tabela 4). Entretanto, itens como áreas de locomoção, iluminação, quarto de dormir, banheiro e escada apresentaram riscos para mais de $50 \%$ da população em estudo. 
Tabela 4 - Principais fatores de risco encontrados nos domicílios de idosos com AVE. Jequié, BA, 2008.

\begin{tabular}{|c|c|c|c|}
\hline Locais & Fatores de Riscos & $\mathrm{n}$ & $\%$ \\
\hline \multirow[t]{2}{*}{ Áreas de locomoção } & Áreas de locomoção impedidas & 12 & 48 \\
\hline & Tapetes não fixos & 21 & 84 \\
\hline \multirow[t]{8}{*}{ Iluminação } & Intensidade baixa principalmente em banheiros e escada & 20 & 80 \\
\hline & $\begin{array}{l}\text { Insuficiente para iluminar toda a superfície da marcha no } \\
\text { interior dos cômodos }\end{array}$ & 13 & 52 \\
\hline & $\begin{array}{l}\text { Não utilização de luzes noturnas e luminárias com bases } \\
\text { seguras }\end{array}$ & 22 & 88 \\
\hline & Interruptores de difícil localização & 11 & 44 \\
\hline & $\begin{array}{l}\text { Ausência de sentinela iluminando quarto, corredor e } \\
\text { banheiro }\end{array}$ & 25 & 100 \\
\hline & Fios dos aparelhos espalhados & 8 & 32 \\
\hline & Insuficiente para iluminar a entrada do domicilio & 20 & 80 \\
\hline & Ausência de luz indireta na cama & 22 & 88 \\
\hline \multirow[t]{4}{*}{ Quarto de dormir } & Guarda-roupa: cabides de difícil acesso & 7 & 28 \\
\hline & Ausência de cadeira permitindo assentar para se vestir & 19 & 76 \\
\hline & Ausência de controle de luz e telefone ao lado da cama & 18 & 72 \\
\hline & $\begin{array}{l}\text { Cama de altura inadequada }(<45 \mathrm{~cm}) \text { ou ausência de } \\
\text { colchão firme }\end{array}$ & 9 & 36 \\
\hline \multirow[t]{5}{*}{ Banheiro } & $\begin{array}{l}\text { Área do chuv eiro com ausência de piso antiderrapante e } \\
\text { cadeira de banho }\end{array}$ & 21 & 84 \\
\hline & Lavabo: difícil acesso ou pouco fixo & 16 & 64 \\
\hline & Inadequação da altura do vaso sanitário & 21 & 84 \\
\hline & Ausência de barras de apoio laterais e paralelas ao vaso & 24 & 96 \\
\hline & Box com abertura difícil e cor tinas instáveis & 18 & 72 \\
\hline \multirow[t]{2}{*}{ Cozinha } & Armários altos com necessidade do uso de escadas & 8 & 32 \\
\hline & $\begin{array}{l}\text { Pia com vazamento e que não permite a entrada de cadeira } \\
\text { de rodas se necessário }\end{array}$ & 11 & 44 \\
\hline \multirow[t]{7}{*}{ Escadas } & Sem revestimento antiderrapante & 22 & 88 \\
\hline & Ausência de interruptores no iní cio e no final da escada & 24 & 96 \\
\hline & $\begin{array}{l}\text { Ausência de identificação nas bordas dos degraus com faixa } \\
\text { amarela }\end{array}$ & 25 & 100 \\
\hline & Ausência de corrimão bilateral e sólido & 24 & 96 \\
\hline & $\begin{array}{l}\text { Ausência de corrimão que se prolongue além do primeiro e } \\
\text { do ultimo degrau }\end{array}$ & 24 & 96 \\
\hline & $\begin{array}{l}\text { Ausência de espel ho de degrau fechado, com lixas } \\
\text { antiderrapantes }\end{array}$ & 25 & 100 \\
\hline & Ausência de uniformidade entre os degraus & 21 & 84 \\
\hline Sala & Móveis dispostos de forma a dificultar a circulação & 9 & 36 \\
\hline
\end{tabular}

Fonte: Dados da pesquisa, 2008 
Nas áreas de locomoção, foram observados tapetes soltos e móveis mal posicionados dificultando a circulação. A iluminação foi considerada inadequada pela baixa intensidade e presença de interruptores de difícil localização. Os banheiros apresentavam pisos escorregadios e ausência de cadeira de banho, elevação do vaso sanitário e barras de apoio.

A escada foi o ambiente de estrutura menos adequada, com ausência de piso antiderrapante, interruptores no início e no final, corrimão bilateral que se prolongue além do primeiro e último degraus, e uniformidade dos mesmos.

Um fator importante foi a ausência em todos os domicílios de sentinela iluminando quarto, corredor e banheiro, marcação com faixa amarela nos degraus e lixas antiderrapantes nos espelhos dos degraus.

Verificou-se uma correlação positiva, estatisticamente significativa $(p=0,039)$, entre o equilíbrio dos idosos acometidos pelo AVE com o ambiente domiciliar.

\section{DISCUSSÃO}

A instalação de patologias que ocasionam redução da capacidade física pode provocar efeitos sobre o controle postural do indivíduo, especialmente as doenças neurológicas que em geral afetam o equilíbrio dinâmico independentemente da topografia lesional. Isso ocorre porque é necessária a interação de vários sistemas múltiplos para a boa funcionalidade do organismo, ou seja, precisam estar preservados os sistemas sensorial, cognitivo e motor. ${ }^{9,23,24}$ Concomitantemente, a presença de um ambiente de risco aumenta o desafio para o idoso, pois este apresenta redução da capacidade de reagir a forças desestabilizantes com reações corretivas. Além disso, os problemas com o ambiente são mais perigosos quanto maior for o grau de vulnerabilidade e instabilidade do idoso., ${ }^{9} 15$

A pontuação em tempo obtida no teste TUG tem forte relação com equilíbrio, velocidade de marcha e capacidade funcional, estando diretamente associada ao nível de mobilidade funcional e à propensão do indivíduo a eventos de quedas. ${ }^{8}$ Entretanto, o corte de tempo para risco de quedas através do teste TUG é variável.

Segundo Shumway-Cook et al., ${ }^{25}$ para idosos saudáveis, foi sugerido que a realização do teste em tempo maior que 14 segundos apresenta alto risco de sofrer quedas, enquanto que Podsiadlo $\&$ Richardson ${ }^{18}$ recomendam que, para idosos portadores de patologias neurológicas, os valores de tempo maiores que 30 segundos são preditores de deficiência de mobilidade e dependência funcional nesta população. Seguindo o corte proposto por Podsiadlo \& Richardson, ${ }^{18}$ adequado para os idosos com AVE do presente estudo, verificou-se que $36 \%$ dos idosos realizaram o teste Timed Up and Go em tempo maior que 30 segundos, apresentando assim, alto risco de sofrer quedas, com mobilidade deficitária e dependência para a maioria de suas atividades de vida diária. ${ }^{18}$

Em um trabalho de Torriani et al., ${ }^{23}$ para verificar comparativamente o desempenho do TUG em pacientes com patologias neurológicas diversas, os portadores de sequelas de AVE apresentaram uma média de 17,88 $( \pm 8,68)$ segundos. No presente estudo, os idosos com AVE apresentaram um menor desempenho do TUG, com média de 28,96 ( $\pm 15,65)$ segundos.

Em relação aos idosos saudáveis residentes na comunidade, estes apresentaram um tempo entre 10,55 a 15,45 segundos de média de desempenho, ${ }^{8,24,26}$ demonstrando médio a alto risco de quedas segundo corte de Shumway-Cook et al. ${ }^{25}$ Comparando estes achados com o tempo de realização do TUG pelos idosos com AVE do presente estudo, pode-se inferir que a patologia neurológica é um fator que influencia no equilíbrio postural, evidenciado pelo menor desempenho no teste de equilíbrio dinâmico.

Quanto à avaliação domiciliar de idosos com sequelas de AVE, verificou-se alta prevalência de risco de quedas nesta população. Este fato somado, às alterações provenientes da patologia 
como perda da função sensoriomotora da extremidade comprometida, presença de convulsões, hemiparesias ou ataxias, predispõe à alta susceptibilidade de quedas dessa população. ${ }^{27}$

Os fatores de risco ambientais de maior relevância encontrados neste estudo estão de acordo com outros artigos encontrados na literatura, ${ }^{7,9,14}$ como iluminação inadequada, superfícies escorregadias, tapetes soltos ou com dobras, degraus altos ou estreitos, obstáculos no caminho e ausência de corrimão em corredores e banheiros.

Por ser o ambiente de maior permanência do idoso, o principal local de ocorrência de eventos de quedas é o próprio domicílio e o risco de sofrer uma queda em casa aumenta com o avançar da idade. ${ }^{28,29}$ Este ambiente pode parecer o mais seguro possível, pelafamiliaridade, porém pode se tornar de alto risco, pois a atenção é reduzida em decorrência da autoconfiança no conhecimento da residência. ${ }^{28}$

A correlação entre equilíbrio e ambiente domiciliar através do TUG e Escala Ambiental de Risco de Quedas Adaptada, demonstrou-se positiva e significante, entretanto, fraca $(p=0,41)$.

Apesar desse fato, há relação entre as variáveis estudadas, levando a acreditar que quanto maior a presença de fatores de risco ambientais e quanto maior o escore do TUG, maior o risco de quedas em idosos com AVE.

\section{CONCLUSÃO}

A dificuldade de equilíbrio apresentada pelos idosos com AVE, associada à alta prevalência de fatores de riscos encontrados no ambiente domiciliar, remete a um quadro preocupante acerca do risco de quedas a que estão sujeitos esses indivíduos, visto que houve correlação positiva entre tais variáveis. Com base nesses achados, torna-se necessária a criação de medidas que visem à prevenção das quedas, atuando nos fatores etiológicos, de caráter intrínseco ou extrínseco, visando a reduzir a incidência desses eventos e as suas consequências, assim como promover a melhoria na qualidade de vida do idoso.

Torna-se pertinente apontar as limitações apresentadas neste trabalho. O risco de quedas é relacionado a uma série de fatores intrínsecos e extrínsecos, que não foram abordados, como: uso de medicamentos, distúrbios visuais, vestibulares ou cognitivos, uso de calçados e vestimentas inadequados no dia a dia, comportamento do idoso frente aos obstáculos, e ambiente externo de risco, ${ }^{14}$ além dos dados não terem sido relacionados com a idade do paciente e o tempo de acometimento pelo AVE. Sugere-se que sejam realizados outros estudos, com maior tamanho amostral, relacionando essas variáveis ao risco de quedas em idosos com alterações físicas provenientes de distúrbios neurológicos, como o AVE. 


\section{REFERÊNCIAS}

1. Lavinsky AE, Vieira TT. Processo de cuidar de idosos com acidente vascular encefálico: sentimentos dos familiares envolvidos. Acta Scientirum Health Sciences 2004; 26(1): 41-5.

2. Somchinda A, Fernandes FC. Saúde e qualidade de vida na Terceira Idade: uma introspecção dos idosos institucionalizados. [monografia] Brasília: ABO; 2003.

3. Souza NR, Oliveira AA; Oliveira MML; Santos CVS; Silva ACC; Sena EL, et al. Olhar sobre o cuidador de idosos dependentes. Revista saúde.com 2005; 1(1): 51-9.

4. Ramos LR. Epidemiologia do envelhecimento. In: Freitas EV, org. et al. Tratado de Geriatria e Gerontologia. Rio de Janeiro: Guanabara Koogan; 2002.

5. Silva F. Acidente vascular cerebral isquêmico prevenção: aspectos atuais - É preciso agir. Medicina interna 2004; 11(2): 99-108.

6. Costa AM, Duarte E. Atividade física e a relação com a qualidade de vida, de pessoas com sequelas de acidente vascular cerebral isquêmico. Revista brasileira de ciencia \& movimento 2002 jan; 10(1): 47-54.

7. Meira EC, et al. Risco de quedas no ambiente físico domiciliar de idosos: Textos Envelhecimento [on-line] 2005. 8(3) [acesso em: 2007 nov 02] 10p. Disponível em: < http:// www.unati.uerj.br/tse/ scielo.php?script $=$ sci_arttext\&pid $=51517$ $59282005000300006 \& \overline{\mathrm{in} g}=\mathrm{pt} \& \mathrm{nrm}=$ iso $>$

8. Guimarães LHCT, et al. Comparação da propensão de quedas entre idosos que praticam atividade física e idosos sedentários. Revista neurociencias 2004; 12(2): 69-73.

9. Fabrício SCC, Rodrigues RAP, Costa Júnior ML. Causas e consequências de quedas de idosos atendidos em hospital público. Rev Saude Publica 2004; 38(1): 93-9.

10. Maciel ACC, Guerra RO. Prevalência e fatores associados ao déficit de equilíbrio em idosos. Revista brasileira de ciencia \& movimento 2005; 13(1): 37-44.

11. Perracini MR, Ramos LR. Fatores associados a quedas em uma coorte de idosos residentes na comunidade. Rev Saude Publica 2002; 36(6): 709-16.

12. Webber AA. Avaliação da propensão a quedas em idosos institucionalizados e nãoinstitucionalizados correlacionando com o nível de cognição e equilíbrio. [monografia] Cascavel: UNIOESTE; 2004.
13. Simoceli L, et al. Perfil diagnóstico do idoso portador de desequilíbrio corporal: resultados preliminares. Rev Bras Otorrinolaringol 2003 nov./dez.; 69(6): 772-7.

14. Sociedade Brasileira de Geriatria e Gerontologia. Projeto Diretrizes sobre quedas em idosos. [on-line]. 2001 [acesso em: 2007 maio 12 ]. Disponível em: URL: http:// www.amb.org.br/projeto_diretrizes/100_diretrizes/QUEDASEM.PDF

15. Santos MLC, Andrade MC. Incidência de quedas relacionada aos fatores de risco em idosos institucionalizados. Revista bahiana de saúde pública 2005 jan-jun; 29(1): 57-68.

16. Organização Mundial de Saúde. Envejecimiento activo: um marco político. Rev Esp Geriatr Gerontol 2002; 37(S2): 74-105.

17. Costa LVA. Política Nacional do idoso: perspectiva governamental. Anais do $1^{\circ}$ Seminário Internacional do Envelhecimento Populacional: uma agenda para o fim do século. Brasília: MPAS, SAS; 1996.

18. Podsiadlo D, Richardson S. The timed up and go: a test of basic mobility for frail persons. J Am Geriatr Soc 1991; 39: 142-8.

19. Secretaria de Estado de Saúde de Minas Gerais. Atenção à saúde do idoso. Belo Horizonte: SAS/MG, 2006.

20. Moraes EN. Protocolo de avaliação multidimensional do idoso. In: Morais EM, organizador. Princípios Básicos de Geriatria e Gerontologia. Belo Horizonte/MG: Coopmed; 2008. p. 157-88.

21. Brasil. Ministério da Saúde. Resolução 196/96 do Conselho Nacional de Saúde/MS sobre Diretrizes e Normas Regulamentadoras de Pesquisa envolvendo seres humanos. Diário Oficial da União, 1996 out 10.

22. Statistical Analysis System - SAS [CD-ROM]. User's guide. Cary: 1999.

23. Torriani C, et al. Avaliação comparativa do equilíbrio dinâmico em diferentes pacientes neurológicos por meio do teste Get Up And Go. Revista neurociencias 2006 jul-set; 14(3): 135-9.

24. Alfieri FM, Teodori RM, Montebelo MIL. Mobilidade funcional de idosos submetidos a intervenção fisioterapêutica. Saúde 2004; 6(14): 45-50.

25. Shumway-Cook A, Brauer S, Woolacott M. Predicting the probability for falls in community- 
dwelling older adults using the Timed Up \& Go Test. Phys ther 2000; 80(9): 896-903.

26. Greve P, et al. Correlações entre mobilidade e independência funcional em idosos institucionalizados e não-institucionalizados.

Fisioterapia em movimento : revista de fisioterapia da PUC-PR 2007 out-dez; 20(4): 117-24.

27. O'Sullivan SB, Schmitz TJ. Fisioterapia: avaliação e tratamento. 2.ed. Barueri/SP: Manole; 2004.
28. Silva TM, et al. A vulnerabilidade do idoso para as quedas: análise dos incidentes críticos. Revista eletronica de enfermagem 2007; 9(1): 64-78.

29. Filgueiras MC, et al. Fraturas em idosos decorrentes de quedas registradas em hospital terciário de referência em traumatologia no ano de 2004. Revista Brasileira de Promoção a Saúde; 20(4): 226-32.

Recebido: 23/10/2008

Revisado: 28/7/2009

Aprovado: 25/8/2009 